\title{
APRESENTAÇÃO: MÉTODOS VISUAIS NA INVESTIGAÇÃO COM PÚBLICOS PLURILINGUES - VISÕES MULTIDISCIPLINARES
}

\author{
Sílvia MELO-PFEIFER \\ Universität Hamburg \\ silvia.melo-pfeifer@uni-hamburg.de \\ https://orcid.org/0000-0002-7371-3293 \\ Tatyana Scheila FRIEDRICH \\ Universidade Federal do Paraná \\ tatyanafriedrich@yahoo.com.br \\ https://orcid.org/0000-0002-9786-7959 \\ Bruna Pupatto RUANO \\ Universidade Federal do Paraná \\ bruna.ruano@gmail.com \\ https://orcid.org/0000-0003-3595-8158 \\ Francisco CALVO DEL OLMO \\ Universidade Federal do Paraná \\ francisco.calvo@ufpr.br \\ https://orcid.org/0000-0003-0139-7639
}

\section{PARA UMA EPISTEMOLOGIA DOS MÉTODOS VISUAIS}

"It is better, we believe, to find new seas on which to sail than old ports at which to dock" ${ }^{1}$ (BARONE \& EISNER, 2012, p. 4)

Os métodos visuais, entre os quais o uso do desenho, são geralmente englobados na designação "arts based research" (BARONO \& EISNER, 2012), "arts based research practice" (LEAVY, 2015) ou ainda "arts-based approaches to inquiry" (TAYLOR, 2010), a par de outras manifestações artísticas como as artes performativas, a escrita criativa ou a 1 "É melhor, cremos, encontrar novos mares para navegar do que velhos portos para atracar"
(tradução nossa).

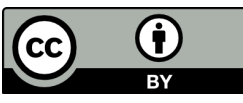


criação de curtas metragens, podendo ser desenvolvida de forma individual ou colaborativa (ANDREWS et al, 2020). Barono \& Einser (2012) defendem que investigação baseada em produção artística se constitui como um esforço para expandir o fazer científico para além dos limites da comunicação verbal, tentando descobrir e reconstruir sentidos que, se limitados à linguagem, continuariam inacessíveis. O que estes diferentes métodos têm em comum é o permitir "to move away from a reliance on language as the main medium and invite[s] attention to modalities such as (...) music, visual arts, dance, theatre and poetry ${ }^{2 "}$ (idem, p. 122). Este afastamento da mediação linguística para construir conhecimento pode justificar-se pelo fato de que a linguagem, não obstante o seu potencial no desenvolvimento da cognição, é sobretudo uma realidade discursiva, mais considerada na investigação pela sua vertente verbal do que não-verbal, tendo assim um alcance limitado quando se trata de representar e/ou reconstruir os sentimentos, as emoções e/ou as experiências traumáticas.

Pela sua natureza, estes métodos enquadram-se predominantemente num paradigma qualitativo de investigação, embora alguns estudos, através da recolha de um número substancial de produções, se possa aproximar do paradigma quantitativo. Por um lado, Leavy esclarece que os métodos artísticos "have posed serious challenges to methods conventions, thus unsettling many assumptions about what constitutes research and knowledge ${ }^{3 "}$ (2015, p. 11), sendo particularmente adequados para desenvolver investigação de teor exploratório, cujas perguntas se centram em processos de descrição, exploração ou descoberta de aspectos da complexa realidade social e da experiência humana. Conforme Barone \& Eisner, "arts based research is a heuristic through which we deepen and make more complex our understanding of some aspects of the world" ${ }^{4}$ (2012, p. 3). Nas palavras de Leavy,

Qualitative research typically claims to be inductive, but can falter with preconceived language, code categories, and guiding assumptions creeping into the process, often more than we may realize. Arts-based practices lend themselves to inductive research designs and the organic emergence of meanings ${ }^{5}(2015$, p. 27).

\footnotetext{
2 "afastar-se da dependência da linguagem como meio principal [de fazer ciência] e dar atenção para modalidades como (...) música, artes visuais, dança, teatro e poesia" (tradução nossa).

3 "têm colocado sérios desafios às convenções sobre métodos, perturbando assim muitos pressupostos sobre o que constitui investigação e conhecimento" (tradução nossa).

4 "a investigação baseada nas artes é uma forma de compreensão através da qual aprofundamos e tornamos mais complexa a nossa compreensão de alguns aspectos do mundo" (tradução nossa).

5 "A investigação qualitativa afirma-se tipicamente como indutiva, mas pode vacilar com linguagem preconcebida, categorias de código, e pressupostos orientadores que se arrastam pelo processo [de investigar], muitas vezes mais do que podemos imaginar. As práticas baseadas nas artes prestamse a desenhos de investigação indutiva e à emergência orgânica de significados" (tradução nossa). 
Pela sua natureza e, sobretudo, pelo rompimento com um paradigma da ciência ancorado em noções de objetividade, replicabilidade e generalização, explicação e certeza, os métodos visuais são um sinal do "postmodernist turn" da investigação, que abraçam a "epistemologia da ambiguidade" (BARONE, 2001, apud TAYLOR, 2010, p. 244; SOUSA SANTOS, 1989), isto é, o reconhecimento de que todo o conhecimento é parcial, incompleto, não generalizável porque local, construído por tentativas, por vezes contraditório e multiditerminado. O uso de métodos visuais e artísticos inscreve-se, pois, num "novo discurso sobre a ciência" (parafraseando o título da obra de Sousa Santos, 1989) que postula "em vez da eternidade, a história; em vez do determinismo, a imprevisibilidade; em vez do mecanicismo, a interpenetração, a espontaneidade e a auto-organização; em vez da reversibilidade, a irreversibilidade e a evolução; em vez da ordem, a desordem; em vez da necessidade, a criatividade e o acidente" (SOUSA SANTOS, 1987, p. 28).

Dentre as qualidades reconhecidas dos métodos visuais, as seguintes poderão ser citadas: a ambiguidade interpretativa dos seus produtos, abrindo espaço ao diálogo e à intersubjetividade; a combinação de elementos linguísticos e não linguísticos, alargando a quantidade e qualidade de meios disponíveis para exploração pelo investigador; a promoção de atitudes e posicionamentos de investigação e de comunicação em ciência mais empáticos para com os participantes na investigação; a possibilidade de aproximar a ciência a outros objetos de investigação e a realidades não linguísticas; a aproximação entre modos de criação de ciência que se aproximam da comunicação em ciência.

\section{OS MÉTODOS VISUAIS NO ESTUDO DO SUJEITO PLURILINGUE}

Os métodos visuais vêm ganhando visibilidade crescente na investigação junto a públicos plurilingues e na formação profissinal, docente ou não (por exemplo: DE LAURENTIS BRANDÃO, 2021; CHIK et al, 2018; KALAJA \& MELO-PFEIFER, 2019; MOLINIÉ, 2009; MOORE \& CASTELLOTTI, 2011). Ainda que se tenham vindo a estabelecer no que Kalaja \& Pitkänen-Huhta (2018) designam por "visual turn" (que também inclui o estudo de paisagens linguísticas ${ }^{6}$; MELO-PFEIFER \& LIMA-HERNANDEZ, 2020), os métodos visuais não implicam a negação da tradição investigativa, metodológica e epistemológica dos estudos em Linguística Aplicada. $\mathrm{Na}$

\footnotetext{
${ }^{6}$ Mencione-se o projeto europeu Erasmus Plus LoCALL (LOcal Linguistic Landscapes for global language education in the school context), coordenado pela Universidade de Hamburgo e desenvolvido entre 2019 e 2022, concebido no quadro de uma educação para o plurilinguismo e para o desenvolvimento da consciência linguística crítica. Disponível em: https://locallproject.eu/ Acesso em: 25 out. 2020.
}

Revista X, v. 16, n. 2, p. 319-332, 2021. 
verdade, eles podem ser vistos em complementaridade com métodos mais estabelecidos (como entrevistas de explicitação) ou como tendo autonomia heurística (ou seja, um significado em si mesmos). Taylor refere-se a esta relação dos métodos artísticos e visuais com a tradição científica nos seguintes termos:

Arts-based approaches to inquiry are not an either-or proposition to traditional research paradigms. Arts-based researchers in language education do no service to themselves to define their methods in opposition to more traditional approaches to inquiry. Rather, the literary, visual, and performing arts offer ways to stretch a researcher's capacities for creativity and knowing, creating a healthy synthesis of approaches to collect, analyze, and represent data in ways that paint a full picture of a heterogeneous movement to improve language education $^{7}$ (TAYLOR, 2010, p. 251).

Quer seja por meio de uso de colagens, fotografia ou desenho, para nomear apenas alguns suportes, os métodos visuais permitem aceder a representações de línguas e culturas, conteúdos disciplinares, e outros. No caso de trabalho com públicos plurilingues, os métodos visuais, que poderíamos cunhar como "arts-based language education research" (acrescentando à tipologia de LEAVY, 2015, p. 5), permitem aceder ainda a representações acerca das próprias vivências de sujeitos plurilingues e dos complexos processos subjacentes ao tornar-se plurilingue. Neste sentido, é uma metodologia indispensável ao "identity work" dos sujeitos participantes, permitindo-lhes refletir acerca de experiências associadas com a diferença, a diversidade, o preconceito (idem, p. 24). Mais ainda: no caso de sujeitos plurilingues que (ainda) não dominam a língua maioritária ou/e de investigação, os métodos visuais permitem construir um espaço de investigação e de intersubjetividade que não passa (apenas) pela expressão linguística (como no caso de entrevistas ou questionários escritos), diminuindo as relações hierárquicas entre investigador e participantes na investigação, produzidas durante situações de comunicação exolingues. Dando uma voz multimodal aos sujeitos plurilingues, os métodos visuais permitem uma aproximação menos "logocentrica" (BLOCK, 2014) ao complexo universo de representações e modos de expressão desses sujeitos. Conforme esclarecem Kalaja

\footnotetext{
7 "As abordagens de investigação baseadas nas artes não são uma alternativa a paradigmas tradicionais de investigação. Os investigadores baseados nas artes no ensino das línguas não prestam qualquer serviço a si próprios definindo os seus métodos em oposição a abordagens mais tradicionais. Pelo contrário, as artes literárias, visuais e performativas oferecem formas de alargar as capacidades de criatividade e conhecimento do investigador, criando uma síntese saudável de abordagens para recolher, analisar e representar dados de forma a pintar um quadro completo de um movimento heterogéneo para melhorar o ensino das línguas" (tradução nossa).
} 
\& Melo-Pfeifer (2019) na apresentação das vantagens do recurso a métodos visuais no estudo do sujeito plurilingue:

\begin{abstract}
It is now acknowledged that each mode of expression has its possibilities but also its limitations: what it might be possible to express verbally may not be possible visually, and the reverse can be the case, too. Furthermore, the modes might at times complement each other. When addressing aspects of multilingualism as subjectively experienced, which as a rule involves emotionally charged events, visual methodologies can be beneficial, especially in cases where the participants have limited literacy skills (e.g. small children or illiterate adults), linguistic problems (i.e. participants not sharing any language with the researcher) and/or psychological problems (participants suffering from post-traumatic stress disorder, e.g. a migrant with a difficult journey behind him or her). On occasions like these, it might be easier for the participants to use visual methodologies than to share experiences verbally or in writing and/or speaking. It is also true that some individuals simply prefer sharing ideas, opinions, experiences, etc. verbally, whereas others would rather do so visually ${ }^{8}(2019$, p. 4)
\end{abstract}

Neste sentido, os métodos visuais são uma abordagem semiótica da construção e constituição dos repertórios linguísticos e comunicativos dos sujeitos participantes nas pesquisas focado na combinação de elementos verbais (eventualmente escritos em diferentes línguas) e não verbais, no que se poderia chamar "translanguaging multimodal" (CHIK \& MELO-PFEIFER, 2020).

Atendendo à tradição do uso de desenhos na investigação (designamente da Psicologia), poder-se-ia afirmar que o desenho do sujeito plurilingue nos oferece as dimensões dos fatos a que atribuem mais significado, a interpretação que fazem dos acontecimentos que observaram ou imaginaram (ex. associações de ideias e de conceitos,

\footnotetext{
8 "Reconhece-se agora que cada modo de expressão tem as suas possibilidades mas também as suas limitações: o que pode ser possível expressar verbalmente pode não ser possível visualmente, e o contrário também pode ser o caso. Além disso, diferentes modos podem por vezes complementar-se uns aos outros. Ao abordar os aspectos do multilinguismo como subjectivamente experimentados, o que geralmente envolve eventos emocionalmente carregados, as estratégias metodológicas visuais podem ser benéficas, especialmente nos casos em que os participantes têm capacidades limitadas de alfabetização (por exemplo, crianças pequenas ou adultos analfabetos), problemas linguísticos (isto é, participantes que não partilham nenhuma língua com o investigador) e/ou problemas psicológicos (participantes que sofrem de transtorno de stress pós-traumático, por exemplo, um migrante com uma viagem difícil atrás dele ou dela). Em ocasiões como estas, poderia ser mais fácil para os participantes utilizar metodologias visuais do que partilhar experiências verbalmente ou por escrito e/ou falando. É também verdade que alguns indivíduos preferem simplesmente partilhar ideias, opiniões, experiências, etc. verbalmente, enquanto outros preferem fazê-lo visualmente" (tradução nossa).
}

Revista X, v. 16, n. 2, p. 319-332, 2021. 
relações entre sujeitos, ...) e os sentimentos e as emoções associados aos acontecimentos/ fenômenos linguísticos experienciados. Especificamente no estudo das crianças plurilingues, o recurso ao desenho serviu para analisar a relação das crianças com as suas línguas, mais precisamente as suas representações acerca:

- de línguas específicas e da diversidade linguística e cultural (ex: estudos em MELO-PFEIFER \& SIMÕES, 2017; SIMÕES, 2006);

- de fenômenos linguísticos (ex: o que é uma língua? PERREGAUX, 2011);

- da aprendizagem de línguas (ex: MOORE, 2005; SIMÕES, 2006);

- do bilinguismo e do plurilinguismo individual (CASTELLOTTI \& MOORE, 2009; KRUMM, 2002; MELO-PFEIFER \& SCHMIDT, 2012; MOLINIÉ, 2009; MOORE, 2005);

$\mathrm{Na}$ formação de professores de línguas, o recurso ao desenho contribuiu para compreender as imagens que os futuros professores têm das línguas que vão ensinar, dos métodos de ensino-aprendizagem e dos públicos escolares (cf. MELO-PFEIFER, 2019, para uma síntese destes estudos), assim como dos processos de desenvolvimento da competência plurilingue (MELO-PFEIFER \& CHIK, 2020).

\section{O PRESENTE NÚMERO TEMÁTICO}

O presente dossiê temático pretende dar conta de investigações empíricas que, partindo de embasamentos disciplinares específicos, tenham feito uso de métodos visuais junto a públicos plurilingues (migrantes e refugiados, aprendentes e professores de línguas ou outros), em diversas áreas disciplinares. O recurso a uma abordagem multidisciplinar justifica-se pela necessidade de interrogar formas de fazer investigação "em piloto automático", ancoradas no habitus investigativo disciplinar e nos seus instrumentos heurísticos canônicos. Através da reprodução de produções visuais dos sujeitos envolvidos na investigação, o leitor tem a sensação de literalmente estar a ver o mundo pelos olhos do sujeito investigado, devido à possibilidade de re-experimentação do real que os métodos visuais proporcionam. Por este motivo, como veremos, muitas vezes o corpus de desenhos pode aparecer ao leitor como pouco acompanhado de argumentos ou explicações. Barone \& Eisner explicam, a este propósito, que o uso de métodos artísticos pode produzir “deep persuasion" (2012, p. 20), em que as palavras poderão parecer supérfluas.

Visto que o número especial apresenta investigações empíricas baseadas no uso sobretudo de desenho como método de geração de dados, convém assinalar que 
the use of drawings to study emotional and cognitive development, trauma and fears, and more recently issues of identity, has a rich story, and using drawings in participatory research with children and young people along with adult groups, such as beginning teachers, is a wellestablished "low-tech" methodology" (Mitchell, 2017, p. 93)

O presente número temático abre com entrevistas de duas reconhecidas especialistas na investigação em Linguística Aplicada com recursos a métodos visuais: as Professoras Paula Kalaja (Universidade de Jyväskylä, Finlândia) e Ana Barcelos (Universidade Federal de Viçosa, Brasil). As duas especialistas discorrem sobre os benefícios do recurso a narrativas visuais (em forma de desenho), as metodologias de análise mais usadas na investigação, as vantagens e também os obstáculos no trabalho com estes métodos. As especialistas refletem acerca das dificuldades em estabelecer os métodos visuais numa Academia marcada pela valorização de dados linguísticos e acerca do potencial mais alargado destes métodos, designadamente como instrumento de ensino, aprendizagem e reflexão na aula de línguas. E há ainda espaço para deixar uma lista de desejos ao gênio da lâmpada...

A contribuição "A biografia linguística visual como instrumento de pesquisa multimodal sobre o desenvolvimento da competência plurilingue", da co-autoria de Ślvia Melo-Pfeifer \& Francisco Calvo del Olmo, da Universidade de Hamburgo e da Universidade Federal do Paraná, respectivamente, reflete acerca do potencial investigativo do recurso a um gênero específico das narrativas visuais: a autobiografia linguística visual. Partindo de uma análise multimodal de 30 daqueles artefatos, recolhidos na Universidade Federal do Paraná, os autores estudam as constelações linguísticas, os atores, tempos e espaços evocados pelos sujeitos para ilustrar o processo de tornar-se plurilingue.

No artigo "Desvelando a paixão pelo ensino de professores de inglês em formação inicial", as autoras Jamylla Barbosa Moreira Silva e Ana Maria Ferreira Barcelos, da Escola Estadual Dr. Raimundo Alves Torres e da Universidade Federal de Viçosa, discorrem acerca de percursos de desenvolvimento profissional de professores estagiários de inglês, num curso de extensão. Combinando três métodos de recolha de dados - questionários, entrevista e narrativas visuais - as autoras ilustram as imagens positivas dos sujeitos em relação à língua objeto de ensino e à profissão, focalizando

\footnotetext{
9 "a utilização de desenhos para estudar o desenvolvimento emocional e cognitivo, traumas e medos, e mais recentemente questões de identidade, tem uma história rica, e a utilização de desenhos na investigação participativa com crianças e jovens, juntamente com grupos de adultos, tais como professores principiantes, é uma metodologia "de baixa tecnologia" bem estabelecida" (tradução nossa).
} 
a emoção "paixão" e a forma como é linguistica e visualmente representada pelos participantes, designadamente a sua rede lexical e semiótica.

João Victor Schmicheck e Amanda Belardo da Silva, no artigo "Drawing the s(elf): Exploring individual repertories through autobiographical illustrations within the English as a lingua franca perspective", analisam, com recurso a desenhos (complementado pela análise de questionários subsequentes), as representações de estudantes do Centro de Línguas e Interculturalidade (Celin) da Universidade Federal do Paraná acerca da língua inglesa, não como língua estrangeira, mas como língua franca. Analisando as produções visuais dos estudantes alusivas ao tema "How did I learn English?", os autores reconstroem as imagens dos estudantes acerca daquela língua e os seus percursos biográficos de aprendizagem, servindo-se do questionário como método adicional de explicitação dos resultados obtidos.

Os três estudos que se seguem, realizados na Universidade Federal do Paraná, "Sensibilização à diversidade linguística através dos métodos visuais: um relato com migrantes e refugiados graduandos da UFPR", "Metodologias visuais na educação em direitos humanos com estudantes migrantes na UFPR", e "Contando Histórias, resgatando memórias: arte como mediadora para o resgate de trajetórias e memórias de crianças migrantes" utilizam os métodos visuais e artísticos junto a população migrante e refugiada, ilustrando o seu potencial pedagógico e multidisciplinar. Os estudos situam-se, respectivamente, nas áreas da Linguística Aplicada, do Direito e da Psicologia.

A primeira delas, "Sensibilização à diversidade linguística através dos métodos visuais: um relato com migrantes e refugiados graduandos da UFPR", da autoria de Bruna Pupatto Ruano e Bruna Faria Martins Francisco, situa-se no âmbito das práticas de sala de aula de Português como língua de acolhimento e com objetivos específicos (acadêmicos) a alunos calouros, e reconstitui a competência plurilingue deste público específico, ao ser-lhes solicitado que desenhassem as línguas que constituem a sua biografia. A par da análise multimodal dos desenhos recolhidos, as autoras lançaram mão das suas notas no momento da apresentação oral dos desenhos pelos estudantes, de forma a acautelar uma maior aproximação ao sentido a eles dados pelos seus autores, não obstante o cunho qualitativo e interpretativo do estudo realizado. Para a análise detalhada, são selecionadas as produções visuais de estudantes originários da Venezuela, Haiti, Síria e República Democrática do Congo, que as autoras relacionam posteriormente com as tendências presentes na totalidade do corpus, colocando em destaque as transversalidades observadas.

Já na contribuição de Tatyana Scheila Friedrich e Jaqueline Bertoldo, situada no contexto da disciplina "Migração, Refúgio e Direitos Humanos", que integra o 
"Programa de Formação Suplementar: Ciclo de Acolhimento Acadêmico para Estudantes Refugiados", do Departamento de Direito da Universidade Federal do Paraná, o enfoque é colocado na experimentação de metodologias diferenciadas no contexto da educação em Direitos Humanos, pelo seu potencial "de co-interpretação e co-construção de sentidos sobre aquilo que envolve o jurídico e os direitos no contexto das migrações e do refúgio no Brasil" (as autoras). Com recurso à análise multimodal de desenhos dos estudantes daquela universidade alusivos ao tema "justiça", acompanhada do recurso às explicações dos seus autores em sala de aula, Friedrich \& Bertoldo identificam as percepções deste público específico acerca das dinâmicas entre migração, Direitos Humanos, justiça e realidade social.

Em "Contando Histórias, resgatando memórias: arte como mediadora para o resgate de trajetórias e memórias de crianças migrantes", as autoras Graziela Lucchesi Rosa da Silva, Camila Akemi Aoto, Luana Lubke, Luisa Martins de Abreu e Lima, Maria Gabriel, Mayssun Omari Osman, Nathalia Tsiflidis e Victória de Biassio Klepa apresentam o projeto de extensão Pequenos do Mundo, da Universidade Federal do Paraná (UFPR). Discutindo a metodologia desenvolvida baseada em atividades artísticas, numa conceção de "arte em suas diferentes expressões e manifestações" útil "para o resgate e compartilhamento das memórias afetivas das crianças e de suas famílias sobre cultura de origem, trajetórias e vivências" (as autoras), esta contribuição aborda a forma como a migração e o refúgio são vivenciadas na infância. Situando-se na área da investigação e intervenção em Psicologia, as autoras explicitam os pressupostos teóricos que embasam as práticas daquela ação e narram os processos de desenvolvimento colaborativo de dois materiais - o Livro de Memórias e a Caixa de Memórias - que se pautam por um princípio comum: o de lutar contra o silenciamento e apagamento da memória, da língua, da cultura e da história a que muitas famílias se vêem sujeitas em situação de migração e refúgio.

Juliane Costa Wätzold, da Universidade de Hamburgo, na contribuição intitulada "Representações de brasilidade nos desenhos e discurso de crianças bilíngues (português/ alemão): um conveniente casamento metodológico", explora o imaginário de crianças bilingues teuto-brasileiras, com recurso à combinação de narrativas visuais e orais, estas últimas geradas por meio de "focus group". Desenvolvido no âmbito de um estudo de cariz etnográfico em contexto de aquisição não-formal do Português, em que o caminho da pesquisa se faz caminhando e não é pré-definido de forma cabal, a autora reflete, num registro próximo da auto-etnografia, acerca das suas opções metodológicas e dos ajustamentos necessários para uma aproximação ao contexto da diáspora e às especificidades do trabalho com públicos infanto-juvenis bilíngues. 
Na contribuição seguinte, Laura Janina Dias Amato, da Universidade Federal da Integração Latino-Americana, explicita os pressupostos teóricos e metodológicos do projeto "Fronteira Intercultural", desenvolvido na rede municipal de Foz do Iguaçu, no contexto de ensino-aprendizagem do Português como Língua Adicional. Neste texto, intitulado "Representação, abordagem intercultural e pedagogia translíngue no Ensino Fundamental I", a autora discorre sobre o potencial transformador de pedagogias plurilingues e interculturais, designadamente sob a ótica decolonizadora das práticas escolares.

A contribuição "Já pode ir?: Primeiras imagens da língua alemã entre estudantes de alemão para fins profissionais", da autoria de Alessandra de Freitas e Carla Alessandra Cursino, centra-se na exploração das imagens que 15 aprendentes em fase inicial da aprendizagem possuem daquela língua. Conforme especificam as autoras, "estes estudantes são, por sua vez, profissionais de enfermagem que estudam o idioma com o objetivo específico de poder exercer sua atividade laboral na Alemanha". A instrução proposta aos alunos foi "de que vocês se lembram ou que associações vocês fazem quando pensam na língua alemã?" e permitiu fazer o levantamento das imagens que os alunos possuem daquela língua (no caso, língua como objeto cultural, objeto sócioeconômico com papel instrumental e objeto afetivo) e observar o que elas podem indicar sobre o processo de aprendizagem por parte daqueles profissionais da saúde. As autoras concluem a sua contribuição sobre o papel das imagens das línguas na aprendizagem e sobre as possibilidades de diversificação dessas imagens.

Finalmente, Carolina Favaretto Santos e Cláudia Cristina Ferreira, da Universidade Estadual de Londrina, propõem uma reflexão sobre a "Apropriação da cultura digital sob a utilização de memes: produzir e ocupar espaços nas redes sociais em prol do ensino de línguas", situando-se no contexto do ensino-aprendizagem on-line em tempos de Pandemia Covid-19. As autoras propõem uma análise multimodal de memes com cariz intercultural e a realização de atividades específicas para as aulas de inglês como língua estrangeira e de português para falantes de outras línguas. As propostas pedagógico-didáticas decorrem da constatação de que os "memes refletem aspectos culturais que marcam determinado momento sócio-histórico e político e identificam comunidades linguístico-culturais ao trazer especificidades e particularidades típicas de cada língua/cultura" (as autoras). Por este motivo, os memes são portadores de um grande potencial intercultural que pode (e deve?) ser explorado na sala de aula, também devido à grande proximidade com os gêneros textuais e discursivos conhecidos dos aprendentes. 
O número especial encerra com a resenha da obra "Comunicación plurilingüe en la educación: Una aproximación etnográfica de la intercomprensión en ambientes universitarios", da autoria de Ángela Erazo, a cargo de Daniel G. G. Sánchez, da Universidade Federal da Paraíba.

Pela sua originalidade, o corpus apresentado nos diferentes artigos e as análises oferecidas aos nossos leitores podem parecer disruptivas, expandindo o que estamos acostumados a aceitar como "dados de investigação" e "provas científicas". Como poderão ver os nossos leitores, o recurso a métodos visuais faz-se de forma isolada (MELO-PFEIFER \& CALVO DEL OLMO) ou combinado com outros métodos de geração e recolha de dados: questionários (SILVA \& BARCELOS; SCHMICHECK \& BELARDO DA SILVA), apontamentos dos investigadores durante as apresentações dos autores dos desenhos (RUANO \& MARTINS; FRIEDRICH \& BERTOLDO) ou entrevistas (COSTA WÄTZOLD). Do ponto de vista de metodologia de análise, o escopo também é diversificado, embora se observe uma tendência para a análise multimodal combinada com análise de conteúdo e das interações. O potencial multidisciplinar do uso de métodos visuais é ilustrado por estudos disciplinarmente situados na Didática de línguas específicas (Inglês, Alemão e Português), no Direito, na Linguística Aplicada (sobretudo ao abarcar temas como a competência plurilingue e as representações linguísticas) e na Psicologia, sendo ainda apresentados estudos ao nível da formação inicial e continuada de professores de línguas e enfermeiros.

Os autores desta apresentação não podem adivinhar o futuro deste volume sobre uma temática que desafia o habitus científico estabelecido, a imaginação científica dos investigadores, ou até as concepções generalizadas do que é plausibilidade, objetividade ou fazer investigação. Ao abraçar este tema, os editores e autores pretendem apresentar novos métodos de aproximação e de explicação das realidades sociais - no caso deste número especial, acerca da aprendizagem de línguas, do plurilinguismo e das mobilidades vividas pelos sujeitos. Trata-se de um objetivo ambicioso já que "as our methods history shows, such debates are critical to scientific progress, as they create a space for a professional public renegotiation of disciplinary practices and standards ${ }^{10 "}$ (LEAVY, 2015, p. 11).

Finalmente, uma segunda ambição: a de chegar a um público leitor mais diversificado do que os que normalmente se interessam pela investigação em Linguística Aplicada, devido ao potencial que os métodos visuais têm na comunicação

\footnotetext{
10 “como mostra a história dos nossos métodos, tais debates são críticos para o progresso científico, ao criarem um espaço para a renegociação pública profissional de práticas e normas disciplinares" (tradução nossa).
}

Revista X, v. 16, n. 2, p. 319-332, 2021. 
da ciência e de que a diversidade de disciplinas representadas neste número dá conta. Com efeito, o uso de métodos visuais constitui-se como uma possibilidade semiótica mais acessível de comunicar os resultados da ciência a uma audiência mais vasta e mais diversa (TAYLOR, 2010).

No decorrer da leitura, os leitores poderão perceber que as imagens foram descritas e optou-se pelo uso de acrescentar uma legenda após cada imagem/figura com a \#PraCegoVer. O intuito é disseminar a cultura da acessibilidade, uma vez que a descrição serve para a apreciação das pessoas com deficiência visual e faz referência ao quanto se utilizam das tecnologias para o desenvolvimento pessoal e social.

\section{REFERÊNCIAS}

ANDREWS, J.; FAY, R.; FRIMBERGER, K.; TORDZYO, G. \& SITHOLÉ, T. Theorising Arts-Based Collaborative Research processes. In: MOORE, E.; BRADLEY, J. \& SIMPSON, J. (eds.), Translanguaging as transformation. The bollaborative construction of new linguistic realities (pp. 118-134). Bristol: Multilingual Matters, 2020.

BARONE, T \& EISNER, E. Arts based research. Los Angeles: SAGE publications, 2012.

BLOCK, D. Moving beyond "lingualism": Multilingual embodiment and multimodality in SLA. In S. May (ed.) The Multilingual Turn. Implications for SLA, TESOL and Bilingual Education, (pp. 54-77). Oxford: Routledge, 2014.

DE LAURENTIS BRANDÃO, A. C. First experiences of teaching EFL in metaphors. Teaching and Teacher Education, 97, 2021. Disponível em: https://doi.org/10.1016/j. tate.2020.103214. Acesso em: 21 fev. 2021.

CHIK, A.; MARKOSE, S. \& ALPERSTEIN, D. (eds.). Languages of Sydney: The people and the passion (143-148). E-Book: Candlin \& Mynard, 2018. URL https://www. candlinandmynard.com/sydney.html.

CHIK, A.; MELO-PFEIFER, S. What does language awareness look like? Visual methodologies in language learning and teaching research (2000 - 2018). Language Awareness, 29, 2020, p. 2-3. https://doi.org/10.1080/09658416.2020.1785481

KALAJA, P. \& MELO-PFEIFER, S. (ed.) Visualising Multilingual Lives More Than Words. Clevedon: Multilingual Matters, 2019.

KALAJA, P. \& PITKÄNEN-HUHTA, A. (eds.) Double special issue 'Visual methods in Applied Language Studies', Applied Linguistics Review 9 (2-3), 2018. 
KRUMM, H.-J. Französisch in den Beinen ... um in die Ferne zu gehen»-Sprachenporträts: ein Zugang zu Sprachbiographien. In: VALS-ASLA, B. 76 (197-202), 2002.

LEAVY, P. Method meets art. Arts-based research practice. New York: the Guilford Press, 2015.

MELO-PFEIFER, S. Comprendre les représentations des enseignants de langues à travers des récits visuels: la mise en images du développement professionnel des futurs enseignants de français langue étrangère. EL.LE, 8(3), 587-610, 2019. http://doi. org/10.30687/ELLE/2280-6792/2019/03/005.

MELO-PFEIFER, S. \& CHIK, A. Multimodal linguistic biographies of prospective foreign language teachers in Germany: reconstructing beliefs about languages and multilingual language learning in initial teacher education. International Journal of Multilingualism, Vol. 17, 2020. Disponível em: https://doi.org/10.1080/14790718.2020.1753748.

MELO-PFEIFER, S. \& LIMA-HERNANDEZ, M. C. "Introdução -Paisagens Linguísticas: ideologias, discursos e práticas multilingues nos espaços sociais". Domínios de Lingu@gem, 14 (4),pp. 1025-1059 2020. URL: http:/www.seer.ufu.br/index.php/ dominiosdelinguagem/article/view/57128/29833.

MELO-PFEIFER, S. \& SCHMIDT, A. Linking "Heritage Language" Education and Plurilingual Repertoires development: evidences from drawings of Portuguese pupils in Germany. In: L1-Educational Studies in Language and Literature, 12 (1-30), 2012. URL $\mathrm{http}: / / 11$.publication-archive.com/public?fn=document\&id=2497\&repository=1.

MELO-PFEIFER, S. \& SIMÕES, A. R. (org.). Plurilinguismo vivido, plurilinguismo desenhado: estudos sobre a relação dos sujeitos com as línguas. E-Book. Colecção "Encontros na Língua Portuguesa". Santarém: Escola Superior de Educação de Santarém, 2017. URL blogs.ua.pt/cidtff/wp-content/uploads/2017/11/livro_metodos_visuais_v3.pdf.

MITCHELL, C. Visual methodologies. In: COE, R.; WARING, M.; HEDGES, L. V. \& ARTHUR, J. (eds.), Research methods \& methodologies in Education (2 $2^{\text {nd }}$ edition) (pp. 92-99), 2017. Los Angeles: SAGE

MOLINIE, M. (eds.). Le dessin réflexif: élément pour une herméneutique du sujet plurilingue. CRTF: Université de Cergy-Pontoise, 2009.

MOORE, D. Rencontres graphiques, pratiques cross-scripturales et mondes imaginés. Synergies France, 4, 2005. URL http://ressources-cla.univ-fcomte.fr/gerflint/France4/ Daniele.pdf.

MOORE, D. \& CASTELlOTTI, V. Dessins d'enfants, recherche qualitative, Revista X, v. 16, n. 2, p. 319-332, 2021. 
interprétation. Des poly-textes pour l'étude des imaginaires du plurilinguisme. In: BLANCHET, PH. \& CHARDENET, P. (eds.). Guide pour la recherche en didactique des langues et cultures. Approches contextualisées (pp. 118-132). Paris : Éditions des archives contemporaines, 2011.

MOSS, J. \& PINI, B. (eds). Visual Research Methods in Educational Research. New York, NY: Palgrave Macmillan, 2016.

PERREGAUX, Ch. Draw me a language! Understanding the Imaginary of Young Children. Child Health and Education, 3(1), 16-30, 2011. URL http://www.childhealthandeducation. com/articles/documents/PerregauxENGLISH31.pdf.

ROSE, G. Visual methodologies. An introduction to researching with visual methodologies. London: Sage, (2016, 4th edition).

SIMÕES, A. R. A cultura linguística em contexto escolar: um estudo no final da escolaridade obrigatória. Tese de Doutoramento. Aveiro: Universidade de Aveiro, 2006. URL https://ria.ua.pt/handle/10773/4681.

SOUSA SANTOS, B. Um discurso sobre as ciências. Porto: Edições Afrontamento. 1987.

TAYLOR, M. C. Arts-based approaches to inquiry in language education. In: KING, K. A. \& HORNBERGER, N. H. (eds.), Encyclopedia of Language and Education, $2^{\text {nd }}$ edition, Volume 10: Research Methods in Language and Education (pp. 243-254). Springer, 2010. 